PROCEEDINGS OF THE

AMERICAN MATHEMATICAL SOCIETY

Volume 130, Number 7, Pages 2001-2005

S 0002-9939(01)06279-7

Article electronically published on November 15, 2001

\title{
ALMOST PERIODIC SOLUTIONS FOR UNDAMPED NONHOMOGENEOUS DELAY-DIFFERENTIAL EQUATIONS
}

\author{
GEORGE SEIFERT
}

(Communicated by Carmen C. Chicone)

\begin{abstract}
We first establish a result giving conditions that certain undamped delay differential equations with almost periodic time dependence have unique almost periodic solutions. Using this result we obtain conditions that a second order scalar nonlinear delay differential equation with almost periodic forcing will have a unique almost periodic solution having saddle-type stability properties. These results use the method of averaging.
\end{abstract}

In a previous paper [1, the author gave conditions that a second order scalar differential equation of the form

$$
x^{\prime \prime}+x-\varepsilon \nu x+\varepsilon^{3} x^{3}=f(t),
$$

where $\varepsilon$ and $\nu$ are positive constants and $f$ is almost periodic (a.p. for short), will have an a.p. solution; cf. also [2]. It was found that this a.p. solution $x(t)$ is for $\varepsilon$ and $\nu$ sufficiently small unique and has saddle point type stability, and in fact $\varepsilon x(t)$ tends to a linear combination of $\cos t$ and $\sin t$ as $\varepsilon \rightarrow 0$ uniformly for $t \in R$, the set of reals. This result was shown to be a special case of a result for a more general first order equation in $R^{n}$ of the form

$$
x^{\prime}=(A+\varepsilon C(t)) x+\varepsilon g(x, \varepsilon)+\varepsilon p(t),
$$

where $A$ and $C(t)$ are real $n \times n$ matrices with $A$ similar to a diagonal matrix with pure imaginary entries, the entries of $C(t)$ are a.p., $g$ and its first partial derivatives with respect to the components of $x$ are continuous in $(x, \varepsilon)$, and $p(t)$ is an $R^{n}$-valued a.p. function. For any $x \in R^{n},|x|$ will denote some norm in $R^{n}$.

Our purpose in this paper is to show that if a time-delay term is introduced into (1), the same result will hold provided the magnitude of this term is sufficiently small, and will follow from a more general $n$-dimensional equation of the form

$$
x^{\prime}=(A+\varepsilon C(t)) x+\varepsilon g(x, \varepsilon)+\varepsilon \alpha h\left(x_{t}\right)+\varepsilon p(t),
$$

where $A, C(t), g$, and $p$ are as before, $\varepsilon$ and $\alpha$ are positive constants, and $h: C_{r} \rightarrow$ $R^{n}$ is a function on $C_{r}$, the set of functions $\phi(\theta)$ continuous on $[-r, \theta]$ to $R^{n}$. For fixed $t$, and an $R^{n}$-valued function $x(t)$ continuous on $R, x_{t}$ is the element of $C_{r}$ given by $x(t+\theta),-r \leq \theta \leq 0$. We use the norm $\|\phi\|_{r}=\sup \{|\phi(\theta)|:-r \leq \theta \leq 0\}$ for $\phi \in C_{r}$. We assume $h$ is uniformly continuous on bounded subsets of $C_{r}$.

Received by the editors February 14, 2000 and, in revised form, January 22, 2001.

2000 Mathematics Subject Classification. Primary 34K14.

(C)2001 American Mathematical Society 
As in 1, we use the method of averaging, but in a way somewhat simpler than the general method of averaging for delay equations discussed in [3] and also [4], in which conditions are required on the spectra of linear operators on $C_{r}$.

As in [1], we make the change of variables $e^{t A} y=x$ in (3) and easily arrive at the equation

$$
y^{\prime}=\varepsilon e^{-t A}\left[C(t) e^{t A} y+g\left(e^{t A} y, \varepsilon\right)+\alpha h\left(e^{A_{t}} y_{t}\right)+p(t)\right]
$$

where $e^{A_{t}} y_{t}=e^{(t+\theta) A} y(t+\theta),-r \leq \theta \leq 0$, is a member of $C_{r}$ for each $t \in R$.

The following lemmas will be proved using essentially the same arguments as were used in the proof of Theorem 1 in 1 . We use the notation

$$
m_{t}(F(t))=\lim _{T \rightarrow \infty} T^{-1} \int_{0}^{T} F(t) d t
$$

for any function $F: R \rightarrow R^{n}$ which is a.p.

Lemma 1. Let $C_{0}=m_{t}\left(e^{-t A} C(t) e^{t A}\right)$,

$$
\begin{aligned}
& g_{0}(y)=m_{t}\left(e^{-t A} g\left(e^{t A} y, 0\right)\right), \\
& b_{0}(\alpha, z)=m_{t}\left(e^{-t A} p(t)+\alpha h\left(e^{A_{t}} z_{t}\right)\right),
\end{aligned}
$$

where $y \in R^{n}$, and $z$ is an a.p. $R^{n}$ valued function. Suppose

$$
C_{0} y+g_{0}(y)+b_{0}(0, z)=0
$$

has a solution $\bar{y}$ such that all the eigenvalues of $C_{0}+\partial g_{0} / \partial y(\bar{y})$ have nonzero real parts; we call such matrices noncritical. Fix $b_{1}>|\bar{y}|$. Then if $z(t)$ is any $R^{n}$-valued a.p. function such that $|z(t)| \leq b_{1}$ for $t \in R$, there exist $\varepsilon_{0}>0$ and $\alpha_{0}>0$ such that if $0<\varepsilon \leq \varepsilon_{0}, 0<\alpha \leq \alpha_{0}$, the equation

$$
y^{\prime}=\varepsilon e^{-t A}\left[C(t) e^{t A} y+g\left(e^{t A} y, \varepsilon\right)+\alpha h\left(e^{A_{t}} z_{t}\right)+p(t)\right]
$$

will, for such a $z(t)$, have a unique a.p. solution $\bar{y}(t, \varepsilon, \alpha, z)$ such that

$$
\bar{y}(t, \varepsilon, \alpha, z) \rightarrow \bar{y} \text { as }(\varepsilon, \alpha) \rightarrow(0,0) \text { uniformly for } t \in R .
$$

Proof. We sketch a proof since it is quite similar to the proof of Theorem 1 in 1]. First, it can be shown that $C_{0}, g_{0}(y)$, and $b_{0}(\alpha, z)$ are well defined since the arguments of $m_{t}$ which define them are a.p., provided $z(t)$ is a.p.; we omit the details. We note that $b_{0}(0, z)$ is independent of $z$ so that $\bar{y}$ is also independent. From the conditions on $C_{0}+\partial g / \partial y(\bar{y})$, it follows that by the Implicit Function Theorem, there exists a $\alpha_{0}>0$ such that if $0 \leq \alpha \leq \alpha_{0}$,

$$
C_{0} y+g_{0}(y)+b_{0}(\alpha, z)=0
$$

has a solution $\bar{y}(\alpha, z)$ such that $\bar{y}(\alpha, z) \rightarrow \bar{y}$ as $\alpha \rightarrow 0$ uniformly for $z(t)$ such that $|z(t)| \leq b_{1}$ where $b_{1}>0$ is as above. For such $z(t)$, it also follows that for $\alpha_{0}$ sufficiently small,

$$
C_{0}+\frac{\partial g_{0}}{\partial y}(\bar{y} \alpha, z)
$$

is noncritical for $0 \leq \alpha \leq \alpha_{0}$.

By the classical (first order) method of averaging (cf. [3]), our lemma will follow as in the proof of Theorem 1 in [1]. We will, however, include some of the details which will be used in the proof of Theorem 1 in this paper. First, there exists an $R^{n}$-valued function $U(t, u, \varepsilon, \alpha, z)$ a.p. in $t$ uniformly for $(u, a, \alpha)$ in compact sets 
of $R^{n} \times(0, \infty) \times[0, \infty]$ and fixed $z(t)$ a.p. in $t$ with $|z(t)| \leq b_{1}, t \in R$, such that the change of variable defined by

$$
y=u+\varepsilon U(t, u, \varepsilon, \alpha, z)
$$

takes $(4 z)$ into

$$
u^{\prime}=\varepsilon f_{0}(u, \varepsilon, \alpha, z)+\varepsilon F(t, u, \varepsilon, \alpha, z)
$$

where $f_{0}(u, \varepsilon, \alpha, z)=C_{0} u+g_{0}(u)+b_{0}(\alpha, z)$ and $F$ satisfies a local Lipschitz condition in $u$, and $F(t, u, \alpha, 0, z)=0$. Since $(4.1 \alpha)$ holds for $y=\bar{y}(\alpha, z), z$ as indicated and $0 \leq \alpha \leq \alpha_{0}$, then if $u=v+\bar{y}(\alpha, z)$, (7) goes into

$$
v^{\prime}=\varepsilon A_{0} v+\varepsilon q(t, v, \varepsilon, \alpha, z)
$$

where $A_{0}=C_{0}+\partial g_{0} / \partial y(\bar{y}(\alpha, z))$, and for $\varepsilon, z$, and $\alpha$ as suitably restricted. Since $A_{0}$ is then noncritical, (8) has a unique a.p. solution $v(t, \varepsilon, \alpha, z) \rightarrow 0$ as $(\varepsilon, \alpha) \rightarrow(0,0)$ uniformly for $t \in R$.

If we denote by $A P$ the set of all a.p. $R^{n}$-valued functions $z(t)$ and use the norm $\|z\|=\sup \{|z(t)|: t \in R\}, A P$ is a Banach space over the reals. Let $A P\left(b_{1}\right)$ be the subset of $A P$ consisting of functions $z(t)$ with $\|z\| \leq b_{1}$. We shall show that if $h$ is Lipchitzian, the mapping $\Phi: A P\left(b_{1}\right) \rightarrow A P\left(b_{1}\right)$ defined by the a.p. solution $y(t, \varepsilon, \alpha, z)$ of $(4 z)$ is a contraction for $\varepsilon$ and $\alpha$ sufficiently small and this will prove

Theorem 1. Assume that the hypotheses of Lemma 1 hold and also that for each bounded set $B \subset C_{r}$ there exists a constant $L(B)>0$ such that for $\phi \in B, \psi \in$ $B,|h(\phi)-h(\psi)| \leq L(B)\|\phi-\psi\|_{r}$. Then there exist $\varepsilon_{0}>0, \alpha_{0}>0$ such that for $0<\varepsilon \leq \varepsilon_{0}, 0<\alpha \leq \alpha_{0}$, (3) has a unique a.p. solution $\bar{x}(t, \varepsilon, \alpha)$ such that $\bar{x}(t, \varepsilon, \alpha) \rightarrow e^{t A} \bar{y}$ as $(\varepsilon, \alpha) \rightarrow(0,0)$ uniformly for $t \in R$; here $\bar{y}$ is as defined in Lemma 1.

Proof. Because of (5) and the condition $b_{1}>|\bar{y}|$, we may assume $\alpha_{0}$ and $\varepsilon_{0}$ so small that $\Phi\left(A P\left(b_{1}\right)\right) \subset A P\left(b_{1}\right), \Phi$ as defined above. Let $z_{i}(t) \in A P\left(b_{1}\right), i=1,2$; let $\bar{u}\left(t, \varepsilon, \alpha, z_{i}\right)$ be the unique a.p. function corresponding to $\bar{y}\left(t, \varepsilon, \alpha, z_{i}\right)$, the unique a.p. solution of $\left(4 z_{i}\right)$ given by Lemma $1, i=1,2$. Let $\bar{v}\left(t, \varepsilon, \alpha, z_{i}\right)$ be the solution of (8) given by $\bar{v}\left(t, \varepsilon, \alpha, z_{i}\right)=\bar{u}\left(t, \varepsilon, \alpha, z_{i}\right)-\bar{y}\left(\alpha, z_{i}\right)$. Then for $\varepsilon_{0}$ and $\alpha_{0}$ sufficiently small, there exists a $\rho, 0<\rho<1$, and a $K>0$ such that

$$
\begin{aligned}
& \left\|\bar{v}_{1}-\bar{v}_{2}\right\| \leq \rho\left\|z_{1}-z_{2}\right\|, \\
& \left\|\bar{u}_{1}-\bar{u}_{2}\right\| \leq\left\|\bar{v}_{1}-\bar{v}_{2}\right\|, \quad \text { and } \\
& \left\|\bar{y}_{1}-\bar{y}_{2}\right\| \leq\left\|\bar{u}_{1}-\bar{u}_{2}\right\|+\varepsilon K\left\|\bar{u}_{1}-\bar{u}_{2}\right\| ;
\end{aligned}
$$

cf. (6). Here we have used the simpler notation $\bar{v}_{i}=\bar{v}\left(t, \varepsilon, \alpha, z_{i}\right)$, etc. So from (8.1)-(8.3) we get

$$
\left\|\bar{y}_{1}-\bar{y}_{2}\right\| \leq \rho\left(1+\varepsilon_{0} K\right)\left\|z_{1}-z_{2}\right\|,
$$

which for $\varepsilon_{0}$ sufficiently small clearly shows that $\Phi$ is a contraction, the unique fixed point of which is an a.p. solution $\bar{y}(t, \varepsilon, \alpha)$ of (4). By our conditions on $A, x=\bar{x}(t, \varepsilon, \alpha)=e^{t A} \bar{y}(t, \varepsilon, \alpha)$ is an a.p. solution of $(3)$.

We now consider the second order scalar equation

$$
x^{\prime \prime}+x-\varepsilon \nu x+\varepsilon^{3} x^{3}-\varepsilon \alpha \ell\left(x_{t}\right)=f(t)
$$


where $\varepsilon, \nu, \alpha$ are positive constants, $f$ is a.p., and let $\ell(\phi): C_{r} \rightarrow R$ be linear on $C_{r}$. Multiplying (1.1) by $\varepsilon$ and putting $\varepsilon x=y$, we get

$$
y^{\prime \prime}+y-\varepsilon \nu y+\varepsilon y^{3}-\alpha \varepsilon \ell\left(y_{t}\right)=\varepsilon f(t) .
$$

Since these equations are perturbations of the corresponding scalar equations in [1], the method to apply Theorem 1 to (1.2) is quite similar to the one used in [1], but we include most of the details here for the sake of clarity.

A system in $R^{2}$ equivalent to (1.2) is

$$
\left(\begin{array}{l}
x_{1} \\
x_{2}
\end{array}\right)^{\prime}=\left(\begin{array}{rr}
0 & 1 \\
-1 & 0
\end{array}\right)\left(\begin{array}{l}
x_{1} \\
x_{2}
\end{array}\right)+\varepsilon\left(\begin{array}{ll}
0 & 0 \\
\nu & 0
\end{array}\right)\left(\begin{array}{l}
x_{1} \\
x_{2}
\end{array}\right)-\varepsilon\left(\begin{array}{c}
0 \\
x_{1}^{3}
\end{array}\right)-\varepsilon \alpha\left(\begin{array}{c}
0 \\
\ell\left(x_{t}\right)
\end{array}\right)+\varepsilon\left(\begin{array}{c}
0 \\
f(t)
\end{array}\right)
$$

where $x_{1}=y, x_{2}=y^{\prime}$.

If we put

$$
\begin{gathered}
x=\left(\begin{array}{c}
x_{1} \\
x_{2}
\end{array}\right), \quad A=\left(\begin{array}{rr}
0 & 1 \\
-1 & 0
\end{array}\right), \quad g=\left(\begin{array}{c}
0 \\
x_{1}^{3}
\end{array}\right), \\
h(\phi)=\left(\begin{array}{c}
0 \\
\ell(\phi)
\end{array}\right), \quad p(t)=\left(\begin{array}{c}
0 \\
f(t)
\end{array}\right), \quad C(t)=\left(\begin{array}{ll}
0 & 0 \\
\nu & 0
\end{array}\right),
\end{gathered}
$$

then (1.3) becomes (3) with $n=2$.

It follows by direct calculation that

$$
\begin{aligned}
C_{0} & =m_{t}\left(e^{-t A} C(t) e^{t A}\right)=\frac{\nu}{2}\left(\begin{array}{rr}
0 & -1 \\
1 & 0
\end{array}\right), \\
g_{0}(x) & =m_{t}\left(e^{-t A} g\left(e^{t A} x\right)\right)=\frac{3}{8}\left(\begin{array}{r}
\left(x_{1}^{2}+x_{x}^{2}\right) x_{2}^{2} \\
-\left(x_{1}^{2}+x_{2}^{2}\right) x_{1}
\end{array}\right), \\
b_{0}(\alpha, z) & =m_{t}\left(e^{-t A}\left(\begin{array}{c}
0 \\
f(t)+\alpha \ell\left(z_{t}\right)
\end{array}\right)\right)=\left(\begin{array}{r}
-b_{1}-\alpha d_{1}(z) \\
b_{2}+\alpha d_{2}(z)
\end{array}\right), \\
\text { where } b_{1} & =m_{t}(f(t) \sin t), b_{2}=m_{t}(f(t) \cos t), \\
d_{1} & =m_{t}\left(\ell\left(z_{t}\right) \sin t, d_{2}=m_{t}\left(\ell\left(z_{t}\right) \cos t\right),\right.
\end{aligned}
$$

and $z(t)$ is any a.p. function such that $|z(t)| \leq b_{1}$ for $t \in R, b_{1}$ as in Lemma 1.

We now use Theorem 1. Condition (4.1) becomes

$$
\begin{aligned}
& -4 \nu x_{2}+3\left(x_{1}^{2}+x_{2}^{2}\right) x_{2}-4 b_{1}=0, \\
& 4 \nu x_{1}-3\left(x_{1}^{2}+x_{2}^{2}\right) x_{1}+4 b_{2}=0 .
\end{aligned}
$$

If $b_{1}=b_{2}=0$, the solution $\left(x_{1} x_{2}\right)=(0,0)$ of $(4.11)$ is not of interest since there $\partial g_{0} / \partial x$ is the zero matrix, and since $C_{0}=\frac{\nu}{2}\left(\begin{array}{rr}0 & -1 \\ 1 & 0\end{array}\right)$ is clearly critical, our theorem does not apply.

Suppose $b_{1}=0, b_{2}>0$ and $\nu>\left(9 b_{2} / 2\right)^{\frac{2}{3}}$. Then by routine analysis (4.11) has a real solution $\left(\bar{x}_{1}, 0\right), \bar{x}_{1}<0,4 \nu / 9<\bar{x}_{1}^{2}<4 \nu / 3$. By direct calculation, it then can be verified that the matrix

$$
C_{0}+\partial g_{0} / \partial y(\bar{x})
$$

with $\bar{x}=\left(\bar{x}_{1}, 0\right)$ has 2 nonzero eigenvalues of opposite sign (cf. [1]) and so is noncritical. So Theorem 1 applies and there exist $\alpha_{0}>0, \varepsilon_{0}>0$ such that (1.3) has an a.p. solution $\left(x_{1}(t, \varepsilon, \alpha), x_{2}(t, \varepsilon, \alpha)\right)$ for $0<\alpha \leq \alpha_{0}, 0<\varepsilon \leq \varepsilon_{0}$, such that $x_{1}(t, \varepsilon, \alpha) \rightarrow \bar{x}_{1} \cos t$ and $x_{2}(t, \varepsilon, \alpha) \rightarrow x_{1} \sin t$ as $(\varepsilon, \alpha) \rightarrow(0,0)$ uniformly for $t \in R$. 
Clearly the cases $b_{2}<0, b_{1}=0$, and $b_{2}=0,\left|b_{1}\right|>0$ can be handled similarly; cf. [1], [2]. We thus have the following

Theorem 2. Let $b_{1} b_{2}=0,\left|b_{1}+b_{2}\right|=\mu>0$. Then if $\nu>(9 \mu / 2)^{\frac{2}{3}}$ there exist $\varepsilon_{0}$ and $\alpha_{0}$ such that for $0<\varepsilon \leq \varepsilon_{0}, 0<\alpha \leq \alpha_{0}$, (1.1) has a unique a.p. solution $x(t, \varepsilon, \alpha)$ such that

$$
\varepsilon x(t, \varepsilon, \alpha) \rightarrow\left(\bar{x}_{1} \cos t+\bar{x}_{2} \sin t\right)
$$

as $(\varepsilon, \alpha) \rightarrow(0,0)$ uniformly for $t \in R$. Here $\bar{x}_{1} \neq 0, \bar{x}_{2}=0$ if $b_{1}=0, b_{2} \neq 0$, and $\bar{x}_{1}=0, \bar{x}_{2} \neq 0$ if $b_{1} \neq 0, b_{2}=0$, and $\left(\bar{x}_{1}, \bar{x}_{2}\right)$ solves (4.11).

In terms of the real Fourier series for $f$,

$$
A_{0}+\sum_{i=1}^{\infty} A_{i} \cos \lambda_{i} t+B_{i} \sin \lambda_{i} t
$$

we can easily obtain the following:

Corollary. If $\lambda_{1}=1, B_{1} \neq 0$, then for $\alpha$ and $\varepsilon$ sufficiently small and $\nu>$ $\left(9\left|B_{1}\right| / 4\right)^{2 / 3}$, (1.1) will have a unique a.p. solution $\bar{x}(t, \varepsilon, \alpha) \rightarrow \bar{x}_{1} \cos t$ as $(\varepsilon, \alpha) \rightarrow$ $(0,0)$ uniformly for $t \in R$.

Other results are also easily obtained. For example, if $\nu=\varepsilon^{-1}$, and $\alpha$ and $\varepsilon$ are sufficiently small, we can infer the existence of a unique a.p. solution of the special case of (1.1):

$$
x^{\prime \prime}+\varepsilon^{3} x^{3}+\varepsilon \alpha \ell\left(x_{t}\right)=f(t) .
$$

We may also assume even in the general case of Theorem 1, that $r=\infty$; i.e., $h(\phi)$ is a function on $C_{\infty}$, the space of functions continuous and bounded on $(-\infty, 0]$ with the usual supremum norm; i.e., the infinite delay case.

An open question is whether $\alpha$ can be deleted from (2) and (1.1) and replaced by some other condition on $h$ in (2) or $\ell$ in (1.1), or in general, whether such results as Theorem 1 can be obtained in cases where the delay term is not small.

Finally, it may be observed that equations such as (1.1) can model a kind of oscillator with a control that depends linearly on the state $y$ not only at time $t$, but at a previous time $t=r$, the delay being a consequence of the fact that the control is situated at a distance from the oscillator. If the forcing is the sum of periodic functions with incommensurate periods, such as, for example, $a \cos t+b \cos \pi t$, it might be of interest to see if for sufficiently small linearities and controls, the oscillator will display an almost periodic time dependence.

\section{REFERENCES}

1. G. Seifert, On almost periodic solutions for undamped systems with almost periodic forcing, PAMS 31(1) (1972), 104-108. MR 44:2989

2. _ Almost periodic solutions by the method of averaging, Lecture Notes in Mathematics 243, Proceedings of Japan-US Seminar on Ordinary Differential and Functional Equations, 1971, 123-133, Springer-Verlag, Berlin. MR 52:14485

3. J.K. Hale, Theory of Functional Differential Equations, Appl. Math. Sci. 3 (1977), SpringerVerlag, N.Y. MR 58:22904

4. S.N. Chow and J. Mallet-Paret, Integral averaging and bifurcation, J. Diff. Eq. 26 (1977), 112-159. MR 58:7718

Department of Mathematics, Iowa State University, Ames, Iowa 50011

E-mail address: gseifert@iastate.edu 